\title{
вмJ Global Health Why patients prefer high-level healthcare facilities: a qualitative study using focus groups in rural and urban China
}

\author{
Yun Liu, ${ }^{1}$ Liwei Zhong, ${ }^{2}$ Shasha Yuan, ${ }^{3}$ Joris van de Klundert ${ }^{4}$
}

To cite: Liu Y, Zhong L, Yuan S, et al. Why patients prefer high-level healthcare facilities: a qualitative study using focus groups in rural and urban China. BMJ Glob Health 2018;3:e000854. doi:10.1136/ bmjgh-2018-000854

Handling editor Nicola Foster

- Additional material is published online only. To view please visit the journal online (http://dx.doi.org/10.1136/ bmjgh-2018-000854).

Received 26 March 2018 Revised 8 August 2018 Accepted 8 August 2018

\section{Check for updates}

(C) Author(s) (or their employer(s)) 2018. Re-use permitted under CC BY-NC. No commercial re-use. See rights and permissions. Published by BMJ.

${ }^{1}$ Erasmus School of Health Policy and Management, Erasmus University Rotterdam, Rotterdam, The Netherlands ${ }^{2}$ Shanghai General Hospital, School of Medicine, Shanghai Jiaotong University, Shanghai, China

${ }^{3}$ Institute of Medical Information and Library, Chinese Academy of Medical Sciences and Peking Union Medical College (IMICAMS), Beijing, China

${ }^{4}$ Prince Mohammad Bin Salman College of Business and Entrepreneurship, King Abdullah Economic City, Saudi Arabia

Correspondence to Yun Liu; liu@eshpm.eur.nl

\section{ABSTRACT}

Introduction Despite policy measure to strengthen and promote primary care, Chinese patients increasingly choose to access higher level hospitals. The resulting overcrowding at higher level hospitals and underutilisation of primary care are viewed to diminish the effects of the continuing health system investments on population health. We explore the factors that influence the choice of healthcare facility level in rural and urban China and aim to reveal the underlying choice processes.

Methods We conducted eight semistructured focus group discussions among the general population and the chronically ill in a rural area in Chongqing and an urban area in Shanghai. Respondents' discussions of (evidence-based) factors and how they influenced their facility choices were analysed using qualitative analysis techniques, from which we elicited choice process maps to capture the partial order in which the factors were considered in the choice process.

Results The factors considered, after initial illness perception, varied over four stages of health service utilisation: initial visit, diagnosis, treatment and treatment continuation. The factors considered per stage differed considerably between the rural and urban respondents, but less so between the general population and the chronically ill. Moreover, the rural respondents considered the township health centres as default and prefer to continue in primary care, yet access higher levels when necessary. Urban respondents chose higher levels by default and seldom moved down to primary care.

Conclusions Disease severity, medical staff, transportation convenience, equipment and drug availability played important roles when choosing healthcare facilities in China. Strengthening primary care correspondingly may well be effective to increase primary care utilisation by the rural population but insufficient for the urban population. The developed four-stage process maps are general enough to serve as the basis for (partially) ordering factors influencing facility level choices in other contexts.

\section{INTRODUCTION}

China has a three-tier hospital system, of which the lowest level (level 1), together with

\section{Key questions}

\section{What is already known?}

- Evidence-based factors from four categories (patient, provider, context and composite factors) determine facility level choice in China.

What are the new findings?

- The identified four stages in the choice processes differed considerably between rural and urban populations, but less between the chronically ill and the general population.

- The rural population appeared to consider township health centres as their default access level, while the default choices of urban respondents lie at the higher levels.

\section{What do the new findings imply?}

- To direct access choices towards primary care facilities, improvements in skills, equipment and drug availability might suffice in rural areas, while substantial changes in advancing primary care capacity and compelling regulatory changes are likely to be required in urban areas.

- The role of the nearest grass-roots healthcare facilities appears to be marginal and worthy of reconsideration by policy makers and other stakeholders.

unrated facilities, forms the primary care system. ${ }^{1-3}$ As there is no formal gatekeeper role, however, patients may access the system at any level and facility of their choice. ${ }^{1}$ Chinese patients often choose to directly access higher level hospitals, thus bypassing primary care facilities. As a result, higher level hospitals are overcrowded, while primary care facilities remain underutilised. ${ }^{4}$ The health reform initiated in 2009 has brought considerable investments to strengthen primary care, and a series of policies aimed at improving the utilisation of the facilities at lower levels. ${ }^{156}$ Still, the number of visits to primary care facilities continues to form a decreasing share of the total number of visits, while the share 
of visits to higher level hospitals continues to increase. ${ }^{7}$ These developments are counter to the Declaration of Alma-Ata, which states that primary care facilities should serve as a first contact and provide access as close as possible to where people live and work. ${ }^{8}$

The lack of efficient utilisation of primary care is seen as a cause for the relatively modest improvements in health outcomes achieved for the Chinese population through the continuous and considerable health system investments made over the last decade. ${ }^{9}$ This especially holds true for rural residents, resulting in worsening disparity in health service access and health outcomes between rural and urban residents. ${ }^{410}$ Further, the overcrowding of higher level hospitals has contributed to deterioration of patient-doctor relationships ${ }^{11} 12$ and quality of care. ${ }^{9}$ Thus, it is important to understand the health-seeking behaviour of the Chinese population, and hence develop measures that can more effectively direct patient flow towards lower levels.

Determinant models form a classical approach to understand decision making in health service utilisation, by identifying the factors (determinants) which influence the choice. ${ }^{13}$ There is a growing body of literature adopting this approach, especially from Western contexts, ${ }^{14-17}$ which includes the well-known Behavioral Model of Health Services Use by Andersen and Davidson. ${ }^{16}$ This model conceptualises access to care in the USA using individual and contextual determinants. ${ }^{16}$ The model can be viewed to be static, as it does not address how the dynamics of disease and health service provisioning influence the choices and choice processes.

Another approach to advance scientific understanding of facility choice is to develop process models which conceptualise patient responses to sickness as a dynamic behavioural process, for example, in the form of a sequence of steps. ${ }^{13} 18$ To the best of our knowledge, however, there is very little empirical research that has validated or adopted either of these models to understand choice of system access level since the new round of healthcare reform in 2009. This holds particularly true for the processes by which Chinese patients choose health system access levels.

As context attributes play important roles in decision making $^{18}$ and the validity of such behavioural models cannot be assumed to remain valid when transferred from one society to another, ${ }^{19}{ }^{20}$ empirical models in the Chinese context are called for. A systematic review of recent empirical research in China to elicit evidence on the determinants of facility level choice identifies four categories of factors influencing choice: patient, provider, context and composite factors. ${ }^{21}$ Whether a patient is classified as 'rural' (as opposed to 'urban') is an example of a patient factor, while travel distance from the patient home to the facility is an example of a composite factor (composed of patient attribute home location and provider attribute facility location).

While there is considerable Chinese evidence to support determinant models, ${ }^{21-24}$ there is little evidence on the choice process. Hence, it appears unknown whether patients consider factors simultaneously and weigh them against each other, or alternatively whether (partial) orders exist in which the factors are considered. The answers to these questions may differ among socioeconomic groups and depend on health conditions. ${ }^{24}{ }^{25}$ These differences regard the set of factors considered, as well as the effect of factors on choice. For the Chinese context, there is evidence that such differences exist between rural residents and urban residents, and between patients with chronic diseases and the general population. ${ }^{21}$ Little is known, however, about how these subpopulations differ in their considerations of these factors. Do they consider different factors, weigh them differently, in a different order or at different occasions? Pursuing these unaddressed directions, our research questions are as follows:

1. What are the factors that influence choice of healthcare facility level for Chinese urban and rural populations, and specifically for the chronically ill?

2. What is the process of decision making in which these factors are taken into account by these Chinese populations?

\section{RESEARCH DESIGN AND METHODS \\ Design}

Qualitative research can provide a vast amount of data to understand behaviours within a certain context and to generate theory. ${ }^{26}{ }^{27}$ Robust qualitative methods are especially useful in health service research to generate rich information on patient preferences and subsequently advance theory. ${ }^{172627}$ Given the explorative nature of the research questions, and the limited scientific understanding of the factors and choice process of healthcare facility level in China, we therefore adopt a qualitative approach. The qualitative method focus group discussion (FGD) is especially effective to answer open research questions as we have formulated. ${ }^{28}{ }^{29}$ FGDs allow respondents to express a variety of viewpoints, while at the same time enabling to interact, for example by reacting to viewpoints of other participants, advancing and refining them, or providing alternatives. ${ }^{28} 30$

We organised two FGDs for each of the four subpopulations considered in the first research question separately: the general population living in an urban area (urban-general), patients with chronic diseases living in an urban area (urban-chronic), the general population living in a rural area (rural-general) and patients with chronic diseases living in a rural area (rural-chronic) (see online supplementary appendix 1 for information on the organisation of the FGDs). From the literature 2831 and experts' opinion, the ideal size of a focus group is four to eight people. Thus, we aimed to recruit seven participants for each group. The following open questions served as starting points for the FGDs:

How do you choose healthcare facilities when you feel ill? What are the factors that you consider when seeking health service? 
While the participants had a break after this open part, the interviewers briefly compared the findings with a list of evidence-based factors ${ }^{21}$ (see online supplementary appendix 2 for an overview of the list of evidence-based factors and short descriptions of each factor). In the second part of the FGD, interviewers addressed evidencebased factors not mentioned before the break in a semistructured manner.

Does factor X influence your facility level choice? If yes, how does it influence your choice?

After these initial questions, the moderators asked follow-up questions to elicit further information and encouraged constructive discussion among participants. For each of the questions and throughout the discussion, the study coordinators explicitly invited every participant to express her or his opinion.

At the end of the FGD, each participant received a small gift (with a value of $¥ 30$, approximately US $\$ 4$ ), as a token of compensation for time. The focus group interviews were conducted in May and June of 2017. All interviews were conducted by the first author, with the support of local health service staff. We report the result on this qualitative study following the Consolidated Criteria For Reporting Qualitative research checklist. ${ }^{32}$

\section{Case study site selection and sampling method}

We selected Nanpeng in Chongqing as rural area. Nanpeng is officially classified as rural ${ }^{33}$ while not too far from higher level facilities for its population to consider them when choosing facilities. As urban area we selected Jiangwan in Shanghai, one of China's largest cities.

As the study targets patients with chronic conditions and the general population, selection of respondents was community-based: participants were selected from the community resident databases (in which prevalence of chronic conditions is registered). We selected respondents who are at least 18 years old, are able to provide information verbally, have health service experience, are permanent residents in the study site, and are involved in choosing health service facilities for one or more of their household members. Absence of chronic conditions was an extra criterion for respondents from the general population. For the groups of patients with chronic conditions, we selected patients with hypertension and/ or diabetes who also met the aforementioned inclusion criteria. Among the set of chronic conditions, diabetes and hypertension are particularly prevalent in China ${ }^{34} 35$ and cause high burden of disease. ${ }^{35-37}$ In addition, treatment and management of these two chronic conditions are prioritised in the current health reform. ${ }^{28}$

The participants were recruited by convenience sampling as is common in conducting FGDs. ${ }^{38}$ However, in addition to the aforementioned individual selection criteria, we purposively recruited the sample to ensure that in each subpopulation group both female and male respondents were presented, and respondents of age below 60 years old were included. The research coordinators contacted the participants by phone and scheduled the group discussions.

The villages where the rural participants lived are scattered within the Nanpeng area. The rural focus groups took place at the Nanpeng Township Health Center. The urban focus groups were conducted in a residential office.

\section{Transcription and analysis}

Before starting the formal analysis, we considered the data collected for each of the four subpopulations: urban-general, urban-chronic, rural-general and ruralchronic. We present the significant differences in the demographic variables between rural and urban respondents determined by Fisher's exact test. In case of inconsistencies between the data collected from the two FGDs per subpopulation, additional FGDs needed to be conducted.

The FGD transcripts were analysed using Atlas.ti. We adopted a framework approach, ${ }^{17}{ }^{39}$ in which the evidence-based factors ${ }^{21}$ served as our initial framework. In alignment with the open questions approach to the FGDs, the category development process included an open coding process and a thematic coding stage. ${ }^{39} 40$ First, factors were openly collected from respondents' verbal description. Next, we considered whether these factors fit into the previously known categories obtained through systematic review of empirical research ${ }^{21}$ but did not impose newly found factors into these categories. In this way, a comprehensive category system was developed. These methods enabled to answer the first research question.

To answer the second research question, we first record for each subpopulation how factor exerted its influence and in what condition. Next, we used process maps to structure these findings for each subpopulation separately. Two authors went back and forth to the findings against the transcripts, as well as the choice process maps, to solidify and refine the identified findings.

Each participant provided anonymous demographic information prior to each discussion.

\section{RESULTS \\ The sample}

Each of the eight focus groups was composed of seven residents, except one rural area focus group which included eight participants. In total, 29 rural respondents and 28 urban respondents participated in the discussions. All rural participants joined the Urban-Rural Residence Basic Medical Insurance scheme (URRBMI), and all urban participants joined the Urban Employee Basic Medical Insurance scheme or the URRBMI. The participants' profile is summarised in table 1 and table 2 . The average education level and family annual income level differed significantly $(\mathrm{p}=0.000)$ between urban and rural respondents. Rural respondents have lower education and annual family income. Urban respondents also had 
Table 1 Participants' profile (number, percentage)

\begin{tabular}{|c|c|c|c|c|c|c|c|c|c|c|c|}
\hline \multirow[b]{2}{*}{ Variable } & \multicolumn{5}{|c|}{ Rural groups } & \multicolumn{5}{|c|}{ Urban groups } & \multirow[b]{2}{*}{$P$ values } \\
\hline & CQ1 & CQ2 & CQ3 & CQ4 & Total (\%) & SH1 & $\mathrm{SH} 2$ & $\mathrm{SH} 3$ & SH4 & Total (\%) & \\
\hline Gender & & & & & & & & & & & 0.96 \\
\hline Female & 5 & 5 & 6 & 5 & $21(72.4)$ & 5 & 5 & 5 & 5 & $20(71.4)$ & \\
\hline Male & 2 & 2 & 1 & 3 & $8(27.6)$ & 2 & 2 & 2 & 2 & $8(28.6)$ & \\
\hline Age & & & & & & & & & & & 0.26 \\
\hline$<30$ & 0 & 0 & 0 & 1 & 1 (3.4) & 0 & 0 & 0 & 0 & 0 & \\
\hline $30-45$ & 0 & 0 & 0 & 1 & $1(3.4)$ & 0 & 0 & 0 & 3 & $3(10.7)$ & \\
\hline $46-60$ & 1 & 3 & 3 & 3 & $10(34.5)$ & 0 & 2 & 2 & 1 & $5(17.9)$ & \\
\hline$>60$ & 6 & 4 & 4 & 3 & 17 (58.6) & 7 & 5 & 5 & 3 & 20 (71.4) & \\
\hline Education level & & & & & & & & & & & 0.000 \\
\hline Primary school or lower & 6 & 5 & 4 & 2 & 17 (58.6) & 0 & 1 & 1 & 0 & $2(7.1)$ & \\
\hline Middle school & 1 & 1 & 3 & 3 & $8(27.6)$ & 3 & 1 & 1 & 0 & $5(17.9)$ & \\
\hline High school & 0 & 1 & 0 & 3 & $4(13.8)$ & 4 & 3 & 3 & 2 & $12(42.9)$ & \\
\hline College or university & 0 & 0 & 0 & 0 & 0 & 0 & 2 & 2 & 5 & $9(32.1)$ & \\
\hline Family annual income $(¥ 10000)^{\star}$ & & & & & & & & & & & 0.000 \\
\hline$<1$ & 0 & 0 & 1 & 0 & $1(3.4)$ & 0 & 0 & 0 & 0 & 0 & \\
\hline $1-5$ & 6 & 7 & 5 & 4 & $22(75.9)$ & 0 & 0 & 0 & 0 & 0 & \\
\hline $6-10$ & 1 & 0 & 1 & 4 & $6(20.7)$ & 5 & 5 & 5 & 1 & $16(57.2)$ & \\
\hline $11-15$ & 0 & 0 & 0 & 0 & 0 & 2 & 1 & 1 & 3 & $7(25.0)$ & \\
\hline $16-20$ & 0 & 0 & 0 & 0 & 0 & 0 & 1 & 1 & 0 & $2(7.1)$ & \\
\hline $21-30$ & 0 & 0 & 0 & 0 & 0 & 0 & 0 & 0 & 3 & $3(10.7)$ & \\
\hline Family size (person(s)) $\dagger$ & & & & & & & & & & & 0.16 \\
\hline 1 & 0 & 0 & 2 & 0 & $2(6.9)$ & 0 & 1 & 1 & 0 & $2(7.1)$ & \\
\hline $2-3$ & 6 & 4 & 4 & 3 & $17(58.6)$ & 6 & 5 & 5 & 4 & $20(71.4)$ & \\
\hline $4-5$ & 0 & 3 & 0 & 2 & $5(3.4)$ & 1 & 1 & 1 & 3 & $6(21.4)$ & \\
\hline$>6$ & 1 & 0 & 1 & 3 & $5(3.4)$ & 0 & 0 & 0 & 0 & 0 & \\
\hline Hospital visit experience & & & & & & & & & & & 0.02 \\
\hline Primary care facilities only & 3 & 3 & 4 & 4 & $14(48.3)$ & 2 & 2 & 2 & 1 & $7(25.0)$ & \\
\hline Higher level facilities only & 0 & 0 & 0 & 0 & 0 & 1 & 1 & 1 & 2 & $5(17.9)$ & \\
\hline Both & 4 & 4 & 3 & 4 & $15(51.7)$ & 4 & 4 & 4 & 4 & $16(57.1)$ & \\
\hline
\end{tabular}

*Family annual income was defined as the total income by all family members that lived together with the participant.

${ }^{\dagger}$ Family size was defined as the total number of family members that lived together with the participant.

$\mathrm{CQ}$, groups organized in Chongqing; CQ1, CQ2, SH1, SH2, chronic groups; $\mathrm{CQ} 3, \mathrm{CQ} 4, \mathrm{SH}$, SH4, general groups; $\mathrm{SH}$, groups organized in Shanghai.

visited higher level facilities more frequently than rural respondents $(\mathrm{p}=0.02)$. As the urban respondents were recruited from the same residential community and the rural respondents from satellite villages of a same town, the distances from home to health facilities are similar among the respondents in each group. However, as

Table 2 Distance from home to facility and average time consumed by transportation

\begin{tabular}{llllll}
\hline \multirow{2}{*}{ Facility } & \multicolumn{2}{l}{ Rural groups } & & Urban groups \\
\cline { 2 - 3 } \cline { 5 - 6 } & Distance $\mathbf{( k m )}$ & Time (min) & & Distance (km) & Time (min) \\
\hline Tertiary hospitals & $55-80$ & 120 & $8-10$ & 50 \\
Secondary hospitals & $20-45$ & 60 & $3-5$ & 35 \\
THC (rural)/CHC (urban) & $3-20$ & 40 & 20 & $<1$ & 15 \\
VC/CHS & $<1$ & 20 & 15
\end{tabular}

$\mathrm{CHC}$, community health center; CHS, community health station; THC, township health centre; VC, village clinic. 
displayed in table 2, travel times of rural respondents to any level of healthcare facility are roughly twice the travel times of their urban counterparts.

\section{The identified factors and the facility selection process}

For each of the four identified subpopulations, the findings from the two FGDs largely overlapped, and we found no inconsistencies, indicating that the FGDs provided rich data. Hence, no additional FGDs were necessary.

Not all previously reported evidence-based factors ${ }^{21}$ were spontaneously mentioned by our respondents, nor considered to be of importance when explicitly mentioned to them. Taking urban and rural respondents together, the analysis revealed 10 factors relevant in the decision making. Brief descriptions of the factors are shown in table 3 . The factor facility design was not previously reported in the literature systematically reviewing the evidence. ${ }^{21}$ Together these 10 factors provide an answer to the first research question. The relevance of each of these factors however varied between urban and rural respondents, as further elaborated when addressing the second research question below.

The answer to the second research question is synthesised in figures 1 and 2, which are choice process maps resulting from analyses of rural and urban FGD data, respectively. The maps distinguish four subsequent process stages. Per stage, respondents considered a different subset of factors. The choice processes can therefore be displayed as decision trees, where each of the nodes reflects the choice decision in the corresponding stage. Possible choices were no treatment, informal care (buying medicine from pharmacies, or self-care such as simple physical treatment), visit primary care facility and visit higher level facility.

The narrative presentation of our research findings is organised based on these process maps. The choice processes of urban and rural respondents differ essentially. While chronically ill patients may encounter different and more frequent choices than the general population, the underlying process differences between the two groups are limited and covered in the narrative.
As a general finding, the three sequential stages in the choice process by rural respondents were initial visit, diagnosis and treatment. Thus, rather than considering the purpose of diagnosis as a choice factor, ${ }^{21}$ our analysis reveals it as a process stage. For urban respondents, an additional fourth stage appeared relevant: treatment continuation.

Whether the patient self-assesses the illness as severe or minor has considerable consequences for the remainder of the choice process and the factors considered. The factor severity clearly has priority over other factors. For ease of presentation, the presentation therefore differentiates depending on the initial self-assessed severity. In later stages, we coalesce these initially distinguished choice maps.

For compactness and ease of exposition, we present the findings on the choice processes as follows. First, we present findings on the factors respondents consider during all choice process stages. Subsequently, we present stage-specific factors, following the choice process stage order. Per stage, we address the differences between rural and urban respondents.

\section{Factors influencing choice at multiple stages. Medical staff}

'Medical staff' was found to be an influential factor throughout all stages, for both populations, and with the same effects. Medical skill and personal relation were the two aspects of medical staff that particularly influenced the choice. Lack of competence (skill) of staff at lower level facilities often caused respondents to choose higher level facilities.

If there are more senior doctors at the township health center, it will be better. Then we do not have to visit the big hospitals outside of the town. (CQ3-1 and 4)

Some respondents even conversely avoided formal care and preferred self-care instead.

What they can do at the village clinic (VC) is just a blood pressure test, without any further medical treatment. I don't go there voluntarily, but only go when they inform

\begin{tabular}{ll}
\hline Table 3 Revealed factors & \\
\hline Factor & Description \\
\hline $\begin{array}{l}\text { Self-assessment of illness } \\
\text { Health literacy }\end{array}$ & $\begin{array}{l}\text { Severity of illness, or if the disease is a special disease. } \\
\text { The ability to understand basic health information and make appropriate decisions. }\end{array}$ \\
\hline Facility design & The layout and complexity of the facility. \\
\hline Service convenience & Service procedure, waiting time or total time consumed for one visit. \\
\hline OOP cost & Out-of-pocket cost per visit. \\
\hline Medical staff & $\begin{array}{l}\text { The attributes that involve medical staff, including their medical skill, seniority of the } \\
\text { medical personnel or patient-doctor relationship. }\end{array}$ \\
\hline Drug & Drug variety and availability. \\
\hline Equipment & The availability of enough medical equipment, especially the advanced equipment. \\
\hline Transportation convenience & Transportation time from home to the facility. \\
\hline Self-evaluated clinical outcomes & Self-evaluated clinical outcomes such as effectiveness or efficacy of care. \\
\hline
\end{tabular}




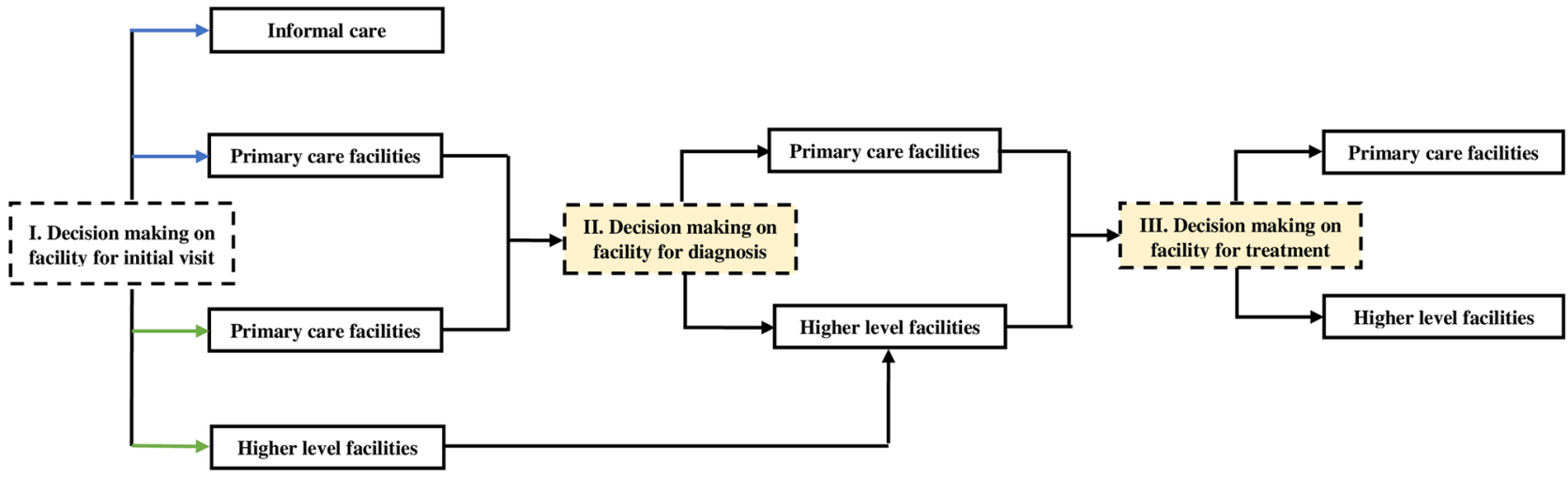

\begin{tabular}{|c|c|c|c|c|}
\hline \multirow{2}{*}{ Factor category } & \multicolumn{2}{|c|}{ I. initial visit } & \multirow{2}{*}{$\begin{array}{c}\text { II. } \\
\text { diagnosis }\end{array}$} & \multirow{2}{*}{$\begin{array}{c}\text { III. } \\
\text { treatment }\end{array}$} \\
\hline & mild & severe & & \\
\hline \multicolumn{5}{|l|}{ Self-assessment of illness } \\
\hline \multicolumn{5}{|l|}{ Health literacy } \\
\hline \multicolumn{5}{|l|}{ Facility design } \\
\hline \multicolumn{5}{|l|}{ Service convenience } \\
\hline \multicolumn{5}{|l|}{ OOP cost } \\
\hline \multicolumn{5}{|l|}{ Medical staff } \\
\hline \multicolumn{5}{|l|}{ Drug } \\
\hline \multicolumn{5}{|l|}{ Equipment } \\
\hline \multicolumn{5}{|l|}{ Transportation convenience } \\
\hline Self-evaluated clinical outcomes & & & & \\
\hline
\end{tabular}

: Decision making point: decision by patients only 1-- Decision making point: decision by patient and professional : Seek care for perceived mild illness — : Seek care for perceived severe/complicated illness : Factor is considered as important

Figure 1 The choice model of healthcare facilities among rural residents. OOP, out-of-pocket.

me of a blood pressure test. When I don't have serious problems for which I need to see doctor, I would rather buy some medicine at the drugstore myself to prevent flu or cold...even though the price is cheaper at VC. (CQ4-1)

Respondents expressed that the more familiar they were with certain medical staff, the more likely they were to choose to visit a facility. For rural respondents, such familiarity appeared to be more likely with lower level staff.
...After a fall at school, my grandson came to the township health center (THC) by himself to receive the treatment! They even didn't require the prepayment for the service and directly gave him treatment...the residents and the staff get along for many years and we are very familiar to each other. (CQ1-5)

Many rural respondents expressed that they felt helpless in higher level hospitals.

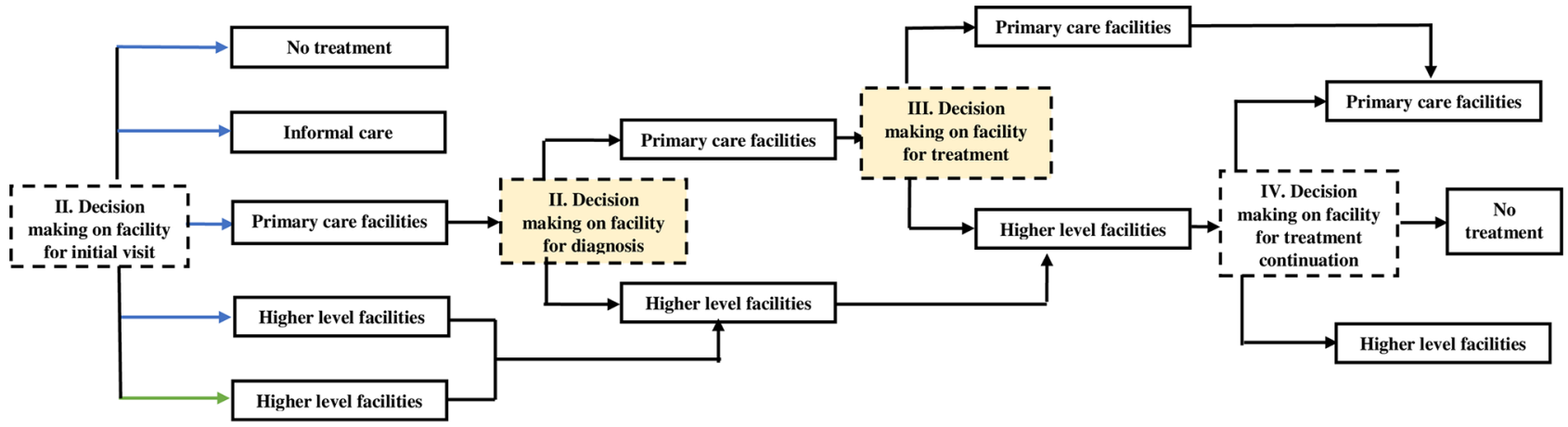

\begin{tabular}{|c|c|c|c|c|c|}
\hline \multirow{2}{*}{ Factor category } & \multicolumn{2}{|c|}{ I. initial visit } & \multirow{2}{*}{$\begin{array}{c}\text { II. } \\
\text { diagnosis }\end{array}$} & \multirow{2}{*}{$\begin{array}{c}\text { III. } \\
\text { treatment }\end{array}$} & \multirow{2}{*}{$\begin{array}{l}\text { IV. treatmen } \\
\text { continuation }\end{array}$} \\
\hline & mild & severe & & & \\
\hline \multicolumn{6}{|l|}{ Self-assessment of illness } \\
\hline \multicolumn{6}{|l|}{ Health literacy } \\
\hline \multicolumn{6}{|l|}{ Facility design } \\
\hline \multicolumn{6}{|l|}{ Service convenience } \\
\hline \multicolumn{6}{|l|}{ OOP cost } \\
\hline \multicolumn{6}{|l|}{ Medical staff } \\
\hline \multicolumn{6}{|l|}{ Drug } \\
\hline \multicolumn{6}{|l|}{ Equipment } \\
\hline \multicolumn{6}{|l|}{ Transportation convenience } \\
\hline Self-evaluated clinical outcomes & & & & & \\
\hline
\end{tabular}

- I: Decision making point: decision by patients only : Decision making point: decision by patient and professional : Seek care for perceived mild illness : Seek care for perceived severe/complicated illness : Factor is considered as important

Figure 2 The choice model of healthcare facilities among urban residents. OOP, out-of-pocket. 
...Everyone in the big hospital is just responsible for her/ his own piece of work. The answers I received most is ' $\mathrm{I}$ don't know' when I looked for my doctor or consulted about something. (CQ1-3)

Urban respondents expressed with much higher volume and richness of information that the familiarity brought three advantages: better quality of care, better service attitude, and lower cost from skipping extra registration fees and tests. It was especially found as a key factor in the stage of treatment continuation for urban respondents, who seek treatment continuation by the same doctor. Likewise, they expressed to highly welcome a policy to facilitate consulting the same doctor for repeat visits.

I am well acquainted with the doctors there (HS hospital). They know everything about me when I step into the hospital...As long as I need to get the medicines, I go to HS hospital...In other hospitals I have to do the blood test and other examinations before I can ask for the drug prescription. In HS hospital I don't have to do those. They know my situation well as a long-term patient for already over 40 years. (SH3-1)

Conversely, they might stop treatment after their doctor has left.

I used to visit Dr. Huang at $\mathrm{CH}$ hospital who was very nice to me and he prescribed the drug for my situation. But later he left the hospital. I don't know where to find him. So, I just stopped taking the drugs and B-scan examination. (SH2-5)

\section{Transportation convenience}

At various stages, transportation convenience acts as a strong factor for both the rural and the urban population, especially at the initial stage for conditions perceived as mild. Rural respondents consider transportation convenience as particularly relevant.

my first option is the township health center because it is close to my home. Only if they cannot handle my situation, then I go to the higher level hospital. (CQ4-1)

\section{Drug and equipment availability}

The availability of drug or equipment strongly influences choice at various stages, for both populations. The unavailability of certain drugs or advanced equipment pushed respondents to higher level facilities, especially in the stage of diagnosis when certain advanced equipment is considered to be required:

...If you have more advanced equipment at the township health center, we will rely on you more...We have no other choice but go to the big hospital. (CQ5-5)

The influence of drug availability was large for urban respondents at the stage of treatment continuation.

...It happens very often that if you want a certain drug, you can only get it from certain tertiary hospitals, or even certain doctors. So, you have to register to visit that doctor, and then get the drug. (SH1-3)
...The drug I need (for cardio disease) is only available at a tertiary hospital and not at the community health center (CHC) or JW hospital (secondary). What is the most annoying, I need 7 types of drugs, but there is a maximum amount of prescription per visit of around 300 Yuan. So, I have to go to the $\mathrm{CH}$ hospital to get the drugs 3 times per 2 weeks. Very annoying! (SH3-3)

Urban respondents are most likely to choose lower level facilities if they have the needed drugs available:

I have special disease. Only if I need the prescription of some specific drugs and they are unavailable at township health center, I go to big hospitals. (CQ1-3)

...If I can get the same drug at lower level, for example the community hospital, then definitely I go there, because it is close to home, and cheaper. (SH4-3)

\section{Factors influencing choice of facility at each stage Initial visit}

The choice of initial facility starts from the self-assessment of illness:

...The sequence of my health seeking behavior is: firstly, I evaluate my illness situation. If the problem is mild, I go to primary level facilities. If it is severe, I go to big hospitals. Among the available big hospitals, I will choose the one that has the expertise to handle my situation and if it is indeed a specific disease that needs treatment within a specialty department, then I go to the big hospital because CHC does not have such special department. (SH1-5)

Subsequently, the decision process differs depending on whether the illness is perceived as minor or as severe/ complicated.

\section{In case of perceived minor illness}

Even in the case of perceived minor illness, respondents worded many factors which caused them not to access the system at the lower, primary level. For instance, both rural and urban respondents were likely to choose self-care instead of formal care, for reasons of service inconvenience and self-evaluated poor clinical outcomes.

I won't bother going to see a doctor if it is just a minor disease such as a cold...I go to drugstore to buy some medicines...the most convenient way. (CQ5-6)

Urban respondents also explained their choice by health literacy.

I think we human beings have self-healing ability...I am afraid of overtreatment very much. When I visit a hospital, there are already a lot of medicines prescribed before I finish reporting my symptoms. Should I take them or not? All the medicines have huge side-effects... (SH4-2)

While most respondents indicated to avoid service at higher level facilities in case of minor illness because of cost and inconvenience, some urban respondents indicated to choose it nevertheless. For them, the higher quality of care outweighed the higher cost of transportation, service and medication, as well as inconvenience of the complex physical environment. 
...I always choose a big hospital when I have health care demand, no matter what is the severity of the problem. Even for teeth extraction, as I trust them and they have better quality. (SH3-5)

\section{In case of perceived severe illness or special care-needed illness}

Urban residents perceive the structural traits associated with big hospitals, such as hospital size and level, as indicators of quality of care. Because of the factor quality of care, they subsequently choose to attend higher level facilities:

... No matter how far away, even by taxi, we go there...for better outcome. (SH4)

Rural respondents consider more factors in case of self-assessed severe illness. Despite the severity, cost and transportation convenience remain key factors that may cause them to choose lower level facilities. Moreover, the complexity of the 'big hospitals' was a negative factor.

...I am always confused by the big hospital's lay out, the electronic display board, and the setting of the department. I hope the local hospital will be better and better, then we don't need to go to the big hospital anymore. (CQ5-6)

\section{Choice of facility for diagnosis}

The default choice after the initial phase is to stay at the same level. Hence, many urban patients will stay at the higher level facilities. It does happen however that patients choose another, typically higher, level for diagnosis. Often such choices were directed by considerations of two factors: medical staff and availability of equipment. When in doubt about the adequacy of medical skills and equipment, patients and doctors usually decide together about switching to a higher level.

\begin{abstract}
...Only when the local hospital cannot handle the condition, for example, problems with the lungs, liver, or cancer, and cannot confirm diagnosis...we have to escalate. (CQ32)
\end{abstract}

...After I got the results from the physical check-up, I asked the doctor at the community hospital. As they couldn't handle it, they advised me to go to the specialized hospital...I usually follow their advice. (SH3-6)

\section{Choice of facility for treatment}

The decision at this stage was usually made by patients and doctors together as well. Urban respondents usually considered self-evaluated clinical outcomes which keep them at or direct them to higher level facilities for treatment.

As long as the problem is solved, I don't care about the cost. (SH3-2)

JW hospital is close by...But because it is not that big, I choose it only for the pre-test and diagnosis. I went to DF hospital (a higher level hospital than JW hospital) instead to receive surgery if needed. (SH2-3)
Rural respondents made trade-offs in the consideration of service price, medical staff and transportation convenience. As a result, they might actually choose to switch towards a primary level facility:

Two years ago I felt uncomfortable and I went to the district hospital first to have a check-up, and the hospital asked me to hospitalize. I was not very willing to stay there because it was too far away from home. So, I called the THC and asked if I could receive the treatment or surgery there. They said yes, so I came back from the high level hospital per my own willingness. (CQ1-5)

\section{Choice of facility for treatment continuation}

In many cases, urban respondents would continue their treatment through repeat visits and/or prescriptions. While many respondents chose to continue at the same facility and level, those who were particularly sensitive to service convenience might choose to switch down from a high-level facility or even stop treatment. There was no mentioning of this stage among the rural respondents.

...At first I got physical tests at XY hospital (a tertiary hospital). For treatment, every time you needed to make a reservation and wait in long queues to get a B-scan, which was so annoying! Later on, I just gave up the test. Just let it go...I don't want to wait after 100 people to get a B-scan. (SH2-1)

\section{DISCUSSION}

In this study, we identified the factors that most influenced the choices of healthcare facility level in rural and urban areas of China and identified the underlying choice processes. To the best of our knowledge, this is the first attempt to present an understanding of these choice processes beyond the identification of factors influencing choice. As the title suggests, it provides qualitative evidence on how patients choose a healthcare facility to access and hence when to bypass nearby primary care facilities. To reduce sample bias, we approached respondents from the general population, instead of from patient populations of certain facilities, as done by most previously reported studies and acknowledged as a limitation. $^{21}{ }^{41}$ By conducting separate and multiple FGDs in rural and urban areas, with respondents from the general population and from the population of patients with chronic conditions, we explicitly distinguished subpopulations which are evidenced to choose differently.

Respondents confirmed the majority of previously reported evidence-based factors. Moreover, the analysis revealed a new factor: facility design. The complexity of tertiary hospitals especially pushed elderly patients towards lower levels.

Respondents considered the factors during a process consisting of four stages: initial visit, diagnosis, treatment and treatment continuation. Respondents were able to provide rich insights into how the factors interact in each of the four stages. The thus arising model of health service behaviour, as grounded in Chinese qualitative evidence, is essentially different from the model proposed by 
Andersen and Davidson ${ }^{16}$ for the US context in two ways. First, the included 'Chinese' factors as confirmed and supplemented by our respondents differ from the factors distinguished by Andersen and Davidson ${ }^{16}$ for the USA. Second, Andersen's model includes choice processes as such but without elaborating them. Our results explicitly address these processes and reveal a staged partial order in which the factors are considered. The four stages resulting from our analysis are quite generic and may validly form a basis to identify the partial order of factors considered in facility (level) choice processes elsewhere.

Among all identified factors, self-assessment of disease severity played a special and important role in the choice process. It served as a prime factor to consider for the phase of initial visit. Other factors only started to weigh in after this self-assessment of severity. As the initially accessed level served as default for the subsequent stages, self-assessment of severity might well be the factor most influential to patient choice. The importance of the initial choice is also reflected in the fact that the number of factors being considered for the initial visit stage is larger than in later stages.

Transportation convenience and medical skill were considered important in all stages. Availability of drugs and equipment had particular large influence on choice at the diagnosis and treatment stages, where they often dominated other factors. Our findings thereby echo a previous study in which urban respondents indicated to prioritise organisational factors,${ }^{41}$ and are also consistent with previous findings that the factor distance is of less importance as illness is more severe. ${ }^{24}$

Some of our findings may reflect initial effects of reform implementation. For instance, the swift referral mechanism was mentioned by multiple urban respondents, and it usually appeared in the decision making during the stage of initial visit or diagnosis. Another example is the long prescription of specific drugs at lower level facilities during the stage of treatment continuation, which was repeatedly confirmed by chronically ill respondents. These findings reflect the effectiveness of medical partnerships, which aim to direct patient flow towards primary care and improve health services utilisation. ${ }^{42}$ More generally, our findings confirm that medical resource sharing through partnerships between facilities of different levels can be effective as it enhances medical skill and equipment in primary care. ${ }^{42}$ Moreover, our findings can help tailor further policy interventions to different subpopulations and process stages.

The choice processes of the rural and urban respondents differed considerably. The rural respondents were much more inclined to access nearby primary care facilities-as intended in the Alma-Ata Declaration ${ }^{8}$ - than their urban counterparts. They considered transportation convenience and cost more, as well as the inconvenience of having to navigate the large hospitals. As a result, primary care often served as the default access level for rural respondents, where they developed relationships with the staff, and they actively considered referral from higher levels to nearby lower level facilities. The default access level was often reserved for the township health centre (THC), rather than the village clinic (VC) - the lower level facility which was usually nearer by. The reasons to seek health services 'higher up' are lack of medical skills and equipment and limitations in drug availability. Policy measures to improve the skills, equipment and drug availability of THCs may therefore enhance their utilisation and exert their potential to act as gatekeepers.

For many urban respondents, the high level 'big hospital' served as the default access level. They developed relationships with the medical staff at these hospitals, and are less affected by distance, travel time or cost barriers, nor are they scared away by the complexity of the higher level urban hospitals. Only the long waiting times and poor attendance pushed them elsewhere, for example, to self-medication. The most significant factor that caused them to choose higher levels was the competence of medical staff. Interestingly, and in contrast to earlier findings, ${ }^{43}$ we found that a large number of respondents valued the patient-doctor relationship over facility-related factors. This suggest that urban respondents may more frequently choose primary care facilities when the physicians with whom they have developed a relationship provide services in primary care facilities as well, and/or when the relationships with the primary care physicians have been improved.

Overall, our results indicate that more substantial changes in advancing primary care capacity and more compelling regulatory changes are required to incentivise the urban population to choose nearby primary care facilities as envisioned in the Declaration of Alma-Ata ${ }^{8}$ and attempted via the ongoing health reforms. ${ }^{3}{ }^{42}$ The growth of the urban population adds further relevance to such policy measures. Let it also be noted that such policy measures may in turn bring (or form) new factors influencing patient choice (eg, comprehensiveness), which will be worthy to be explicitly included in future research.

The differences between Chinese rural and urban residents confirm existing literature, ${ }^{24434}$ and can be due to differences in income, education, health literacy, travel distance to higher level facilities and to the relative importance attached to quality of care. ${ }^{20}{ }^{45}$ In addition, we did not find the stage of treatment continuation in the choice process of rural respondents, perhaps because they are less likely to choose long-term treatment at higher level facilities anyway due to spatial access disparity. ${ }^{46}$

Despite numerous differences between rural and urban, a common result was that the rural focus groups made very little mention of VCs, whereas the urban focus groups never even mentioned the urban equivalent of the community health station (CHS). The role of these nearest grass-roots institutions appears to be marginal and appears to be worthy of reconsideration by policy makers and other stakeholders.

Both rural and urban respondents mentioned self-care in the form of purchasing drugs without prescription 
from a pharmacy to adequately balance time and costs. It confirms the potential for pharmacies to play a role in addressing the health system pressures reported in the literature..$^{22} 47$

Chronic patients may make more intensive use of health service facilities, and typically consider treatment continuation decisions (the fourth stage), whereas the general population might more frequently consider initial visits and diagnosis (the first or second stage). Our results indicate, however, that in a same stage (eg, diagnosis), the factors considered differ little between the general population and the chronically ill. The only difference occurred for urban respondents in the treatment continuation stage, for whom the factor drug availability stood out. This may well be related to financial consequences, reimbursement policies and prescription (in) conveniences for long-term medication, as extensively addressed in the literature on the ongoing health reform. ${ }^{342} 48$

This study had some limitations. Even when considering that China is too large to be fully covered when collecting data, a main limitation of our study is that data collection is only from two study sites. While the consistency of the FGD data confirms internal validity, external validity needs further research. Furthermore, the sampling methods implied participants were mostly female and elderly. Moreover, sampling of chronically ill was restricted to patients suffering from at least one of the two conditions: hypertension and diabetes. While the samples thus include prioritised patient populations, further research may strive to more explicitly address other subpopulations, for example, to more broadly cover the chronically ill. Progressing along these paths, our initial insights in and modelling of the choice processes of the Chinese populations can be improved, extended, refined and updated. Moreover, now that the factors and processes are better understood and mapped, future quantitative research into the factors is called for to clarify the trade-offs and enable effective policy making in relevant contexts. Finally, we suggest further research to address revealed preferences, as opposed to the stated preferences we have collected, to strengthen the evidence base.

\section{CONCLUSION}

Besides individual disease severity and transportation convenience, the organisational factors of healthcare facilities, specifically medical staff, drug and equipment availability, are important in the healthcare facility choice process of rural and urban respondents. The role of the nearest grass-roots institutions (VC and CHS) appears to be marginal and appears to be worthy of reconsideration by policy makers and other stakeholders. For the rural population, our results suggest that policy measures to improve the skills, equipment and drug availability of THCs, and promoting medical resource sharing by medical partnership, may be effective in incentivising the rural population to use primary care facilities. These measures are in line with the ongoing reform. More substantial changes in advancing primary care capacity and compelling regulatory changes are likely to be required to direct access choices of the urban population towards nearby primary care facilities.

The novel four-stage model to describe the health system access choice processes appears general enough to serve as the basis for (partially) ordering factors influencing facility level choices in other contexts.

Acknowledgements We would like to thank Professor Wan Guohua (data collection), Professor Wang Fang (data collection) and Dr Jeroen van Wijngaarden (study design and analysis) for their help and support. We also acknowledge assistance from Bin Hu and Yulan Pu in organising and conducting the focus group discussions

Contributors YL and JvdK conceived and designed the study. LZ and SY coordinated the fieldwork. YL conducted the fieldwork, did the initial analyses of the data and wrote the draft manuscript. LZ and SY commented on the interpretation of the data. YL and JvdK revised and reviewed the manuscript. All authors have seen and approved the final version of the abstract for publication.

Funding This work was partly supported by the China Scholarship Council (CSC) (grant number 201507720036).

Competing interests None declared.

\section{Patient consent Obtained.}

Ethics approval This study received ethical approval from Shanghai General Hospital Medical Ethical Review Committee (no 2017KY207).

Provenance and peer review Not commissioned; externally peer reviewed.

Data sharing statement No additional data are available.

Open access This is an open access article distributed in accordance with the Creative Commons Attribution Non Commercial (CC BY-NC 4.0) license, which permits others to distribute, remix, adapt, build upon this work non-commercially, and license their derivative works on different terms, provided the original work is properly cited, appropriate credit is given, any changes made indicated, and the use is non-commercial. See: http://creativecommons.org/licenses/by-nc/4.0/

\section{REFERENCES}

1. Eggleston K. Health Care for 1.3 Billion: an overview of China's Health System. SSRN Electronic Journal 2012:2-28.

2. National Health and Family Planning Commission of the People's Republic of China. What services can be provided by the primary health care institutions. 2009. http://www.nhfpc.gov.cn/mohzcfgs/ s9664/200904/39933.shtml (accessed 18 Jun 2018).

3. Li X, Lu J, Hu S, et al. The primary health-care system in China. The Lancet 2017;390:2584-94.

4. Wu D, Lam TP. Underuse of Primary Care in China: the scale, causes, and solutions. J Am Board Fam Med 2016;29:240-7.

5. Chen Z. Launch of the health-care reform plan in China. The Lancet 2009;373:1322-4.

6. Yip W, Hsiao W. Harnessing the privatisation of China's fragmented health-care delivery. Lancet 2014;384:805-18.

7. National Health and Family Planning Committee. Statistical yearbook for health and family planning in China. 2017.

8. Anon. Primary Health Care: declaration of Alma-Ata. The Lancet 1978;312:1040-1.

9. Zhang L, Cheng G, Song S, et al. Efficiency performance of China's health care delivery system. Int J Health Plann Manage 2017;32:254-63.

10. Zhai S, Wang P, Dong Q. A study on the equality and benefit of China's national health care system. Int J Equ Health 2017:1-11.

11. Zhao L, Zhang X-Y, Bai G-Y, et al. Violence against doctors in China. The Lancet 2014;384:744.

12. Anon. Violence against doctors: Why China? Why now? What next? Lancet 2014;383:1013.

13. Kroeger A. Anthropological and socio-medical health care research in developing countries. Soc Sci Med 1983;17:147-61. 
14. Ikumi M, LE H, Anh Q. Patient choice of healthcare facilities in the central region of Vietnam. J Int Coop Stu 2017;25:47-64.

15. Grundy J, Annear P. Health-seeking behaviour studies:a literature review of study design and methods with a focus on Cambodia. Health Policy and Health Finance Knowledge Hub 2010;7:1-14.

16. Andersen RM, Davidson PL. Improving access to care in america: individual and contextual indicators. In: Changing the US health care system: key issues in healthservices policy and management, 2007: 3-31.

17. Sobo EJ, Seid M, Reyes Gelhard L, Reyes G. Parent-identified barriers to pediatric health care: a process-oriented model. Health Serv Res 2006:41:148-72.

18. Chrisman NJ. The health seeking process: an approach to the natural history of illness. Cult Med Psychiatry 1977;1:351-77.

19. Ward $\mathrm{H}$, Mertens TE, Thomas $\mathrm{C}$. Health seeking behaviour and the control of sexually transmitted disease. Health Policy Plan 1997:12:19-28.

20. Olenja J. Health seeking behaviour in context. East Afr Med J 2003;80:61-2.

21. Liu Y, Kong Q, Yuan S, et al. Factors influencing choice of health system access level in China: a systematic review. PLoS One 2018;13:e0201887.

22. Yuefeng L, Keqin R, Xiaowei R. Use of and factors associated with self-treatment in China. BMC Public Health 2012;12:1.

23. Gong $\mathrm{CH}$, Kendig $\mathrm{H}, \mathrm{He}$ X. Factors predicting health services use among older people in China: an analysis of the China Health and Retirement Longitudinal Study 2013. BMC Health Serv Res 2016;16:63.

24. Qian D, Pong RW, Yin A, et al. Determinants of health care demand in poor, rural China: the case of Gansu Province. Health Policy Plan 2009;24:324-34.

25. Victoor A, Delnoij DM, Friele RD, et al. Determinants of patient choice of healthcare providers: a scoping review. BMC Health Serv Res 2012;12:272.

26. Bradley EH, Curry LA, Devers KJ. Qualitative data analysis for health services research: developing taxonomy, themes, and theory. Health Serv Res 2007:42:1758-72.

27. Pope C, Ziebland S, Mays N. Qualitative research in health care. Analysing qualitative data. BMJ 2000;320:114-6.

28. Kitzinger J. Qualitative research. Introducing focus groups. BMJ 1995;311:299-302.

29. Kitzinger J. The methodology of Focus Groups: the importance of interaction between research participants. Sociology of Health and Illness 1994:16:103-21.

30. Dicicco-Bloom B, Crabtree BF. The qualitative research interview. Med Educ 2006;40:314-21.

31. McLafferty I. Focus group interviews as a data collecting strategy. $J$ Adv Nurs 2004;48:187-94.

32. Tong A, Sainsbury P, Craig J. Consolidated criteria for reporting qualitative research (COREQ): a 32-item checklist for interviews and focus groups. International Journal for Quality in Health Care 2007:19:349-57.

33. Administrative Planning of Banan District, 2016. People's government of Banan District. http://www.cqbn.gov.cn/bnkq.asp? id=6360 (accessed 20 Dec 2017).

34. Li H, Oldenburg B, Chamberlain C, et al. Diabetes prevalence and determinants in adults in China mainland from 2000 to 2010: a systematic review. Diabetes Res Clin Pract 2012;98:226-35.

35. Gao Y, Chen G, China National Diabetes and Metabolic Disorders Study Group. Prevalence of hypertension in study. PLoS One 2013;8:e65938

36. Yang G, Wang Y, Zeng $Y$, et al. Rapid health transition in China, 1990-2010: findings from the global burden of disease study 2010 . Lancet 2013;381:1987-2015.

37. Forouzanfar MH, Liu P, Roth GA, et al. Global burden of hypertension and systolic blood pressure of at least 110 to $115 \mathrm{~mm} \mathrm{Hg}, 1990$ 2015. JAMA 2017;317:165-82.

38. Marshall MN. Sampling for qualitative research sample size. Family Practice 1996;13:522-5.

39. Suurmond J, Rosenmöller DL, El Mesbahi H, et al. Barriers in access to home care services among ethnic minority and Dutch elderly--a qualitative study. Int J Nurs Stud 2016;54:23-35.

40. Macfarlane A, O'Reilly-de Brún M. Using a theory-driven conceptual framework in qualitative health research. Qual Health Res 2012;22:607-18.

41. Wu D, Lam TP, Lam KF, et al. Health reforms in china: the public's choices for first-contact care in urban areas. Fam Pract 2017;34:cmw133-200.

42. The State Council. 2017. Guiding opinions of the general office of the state council on promoting the construction and development of medical partnership. http://www.gov.cn/zhengce/content/2017-04/ 26/content_5189071.htm (accessed 18 Jun 2018).

43. Wu D, Lam TP, Lam KF, et al. Challenges to healthcare reform in China: profit-oriented medical practices, patients' choice of care and guanxi culture in Zhejiang province. Health Policy Plan 2017;32:1241-7.

44. Wang X, Birch S, Ma H, et al. The structure and effectiveness of health systems: exploring the impact of system integration in rural China. Int J Integr Care 2016;16:1-12.

45. Sun X, Shi Y, Zeng Q, et al. Determinants of health literacy and health behavior regarding infectious respiratory diseases: a pathway model. BMC Public Health 2013;13:261.

46. Wang X, Yang H, Duan Z, et al. Spatial accessibility of primary health care in China: a case study in Sichuan Province. Soc Sci Med 2018;209:14-24.

47. Li J, Shi L, Liang H, et al. Urban-rural disparities in health care utilization among Chinese adults from 1993 to 2011. BMC Health Serv Res 2018:18:1-9.

48. Gao W, Chen B. and Effect of 'TricolorLadder' long-prescription management mode in community. Chinese Gen Pract 2016;19:757-61. 